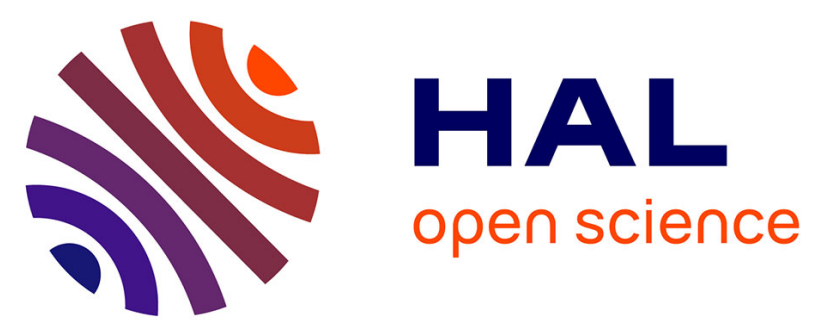

\title{
Capillary Dipoles: Towards Thermocapillary Micromanipulation of Multiple Particles Floating at the Free Surface
}

\author{
Ronald Terrazas, Adrien de Maeijer, Aude Bolopion, Michaël Gauthier, \\ Michel Kinnaert, Pierre Lambert
}

\section{To cite this version:}

Ronald Terrazas, Adrien de Maeijer, Aude Bolopion, Michaël Gauthier, Michel Kinnaert, et al.. Capillary Dipoles: Towards Thermocapillary Micromanipulation of Multiple Particles Floating at the Free Surface. International Conference on Manipulation, Automation and Robotics at Small Scales, Jul 2018, Nagoya, Japan. 10.1109/MARSS.2018.8481155 hal-02927294

\section{HAL Id: hal-02927294 \\ https://hal.science/hal-02927294}

Submitted on 1 Sep 2020

HAL is a multi-disciplinary open access archive for the deposit and dissemination of scientific research documents, whether they are published or not. The documents may come from teaching and research institutions in France or abroad, or from public or private research centers.
L'archive ouverte pluridisciplinaire HAL, est destinée au dépôt et à la diffusion de documents scientifiques de niveau recherche, publiés ou non, émanant des établissements d'enseignement et de recherche français ou étrangers, des laboratoires publics ou privés. 


\section{Capillary Dipoles: Towards Thermocapillary Micromanipulation of Multiple Particles Floating at the Free Surface}

\author{
Ronald Terrazas \\ TIPs Dpt, Université libre de Bruxelles \\ 50 avenue FD Roosevelt, B-1050 Brussels \\ ronald.terrazas@ulb.ac.be
}

\author{
Adrien De Maeijer \\ TIPs Dpt, Université libre de Bruxelles \\ 50 avenue FD Roosevelt, B-1050 Brussels
}

\author{
Aude Bolopion \\ AS2M dpt, FEMTO-ST \\ 24 Rue Alain Savary \\ F-25000 Besançon \\ aude.bolopion@femto-st.fr
}

\author{
Michaël Gauthier \\ AS2M dpt, FEMTO-ST \\ 24 Rue Alain Savary \\ F-25000 Besançon \\ michael.gauthier@femto-st.fr
}

\author{
Michel Kinnaert \\ SAAS Dpt, Université libre de Bruxelles \\ 50 avenue FD Roosevelt, B-1050 Brussels \\ michel.kinnaert@ulb.ac.be
}

\author{
Pierre Lambert \\ TIPs Dpt, Université libre de Bruxelles \\ 50 avenue FD Roosevelt, B-1050 Brussels \\ pierre.lambert@ulb.ac.be
}

\begin{abstract}
This paper presents a contribution to thermocapillary micromanipulation of floating particles at the free surfaces. Based on a physical model for the so-called capillary dipole interaction, the force between two floating particles is predicted with a Bessel function, whose magnitude can be calculated from the measured distance between particles. This force is then considered as a disturbance force and rejected in the control scheme thanks to a feedforward strategy. We provide theoretical results for this strategy as well as an experimental proof of concept.
\end{abstract}

Index Terms-Thermocapillary micromanipulation, capillary forces, optofluides, feedforward, capillary dipole, Cheerios effect

\section{INTRODUCTION}

Within the framework of non-contact micromanipulation, this paper presents a new strategy towards handling multiple particles floating at the free surface thanks to the so-called thermocapillary micromanipulation technique.

Non-contact micromanipulation uses remote force fields to control the position of the objects, such as for instance magnetophoresis [1], dielectrophoresis [2], acoustophoresis [3] or optical effects [4]. However, the manipulation in parallel of several particles independently is a challenge since all the objects are affected by the remotely induced force fields. Different approaches have been proposed, such as anchoring the objects that should not move [5], controlling the force using objects with slightly different physical properties [6], using the noise inherent to the micromanipulation systems [7] or shaping the force field [8], [9].

More recently, some authors have proposed to use the socalled thermocapillary effect. This consists in generating a temperature gradient along the interface between two fluids, leading to a surface stress thanks to the so-called Marangoni effect (surface tension depends on temperature). This stress is then used to trigger flows able to displace particles. In [10][12], $1-100 \mu \mathrm{m}$ particles are moved in the bottom of a water reservoir by heating the top free surface with a laser. In [13], the generation of bubbles in oil is controlled, which in turn creates flows in the oil, able to move particles. A similar approach was applied to bubbles in water [14].

Comparing all these principles, [15] showed the micromanipulation of particles with the laser-induced thermocapillary convection to be an alternative method with less or different drawbacks w.r.t. other non-contact micromanipulation techniques. Especially, four key points can be emphasized: (1) using laser heating to generate thermocapillary convection allows to generate fast localized flows which allows to exert significant forces on the particles and also opens the possibility to perform manipulation in parallel; (2) particles manipulated at the interface (i.e. the free water-air surface) move faster than particles manipulated in the fluid bulk. However, this imposes wettability conditions for the particles to float and remain at the fluid interface; (3) using a closed-loop controller allows to control the unstable system defined when manipulating particles at the interface and also improves the performance of the system; (4) the method is based on a local effect, which opens the way towards multiple particles handling.

However, multiple particles handling remains challenging because of the capillary interactions between them (known as the Cheerios effect). This paper proposes a strategy to reject the capillary perturbation induced by a neighboring particle thanks to a feedforward control scheme. It is structured as follows. Section III presents the physics of lateral capillary forces, Section III recalls the feedback controller presented in [15] and presents the new feedforward controller of this study. Simulation and experimental results are reported in Section IV and discussed in Section $\mathrm{V}$ 


\section{LATERAL CAPILlary ForCeS}

As shown in Fig. 1. lateral capillary forces acting on floating particles can be attractive or repulsive, according to the sign of the capillary dipoles $Q_{i}=r_{i} \sin \phi_{i}$.

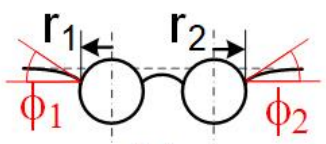

(a)

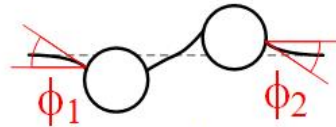

(b)
Fig. 1: Lateral capillary forces between particles [16]. a) Attracted particles with same wettability $\left(r_{1} \sin \phi_{1} \cdot r_{2} \sin \phi_{2}>0\right) \quad$ (b) Repelled particles with opposite wettability $\left(r_{1} \sin \phi_{1} \cdot r_{2} \sin \phi_{2}<0\right)$.

Kralchevsky [16] proposed a model for the lateral capillary force $F_{\text {LatCap }}$, which can be used in an appropriate controller:

$$
F_{\text {LatCap }}=2 \pi \sigma Q_{1} Q_{2} L_{\text {cap }}^{-1} K_{1}\left(L_{\text {cap }}^{-1}\left\|\mathbf{X}_{\text {part 1 }}-\mathbf{X}_{\text {part 2 }}\right\|\right)
$$

where $\sigma$ is the surface tension, $L_{\text {cap }}$ is the capillary length $\sqrt{\frac{\sigma}{\rho_{\text {fluid }} g}}(\mathrm{~m})$ and $K_{1}$ is the modified Bessel function of the second kind, first order. The operator $\|\mathbf{X}\|$ refers to the norm of vector $\mathbf{X}$. One of the assumptions here is that particles should not be very close to each other (typically not closer than $\left.L_{\text {cap }}\right)$.

Equation (1) was experimentally validated using the experimental setup shown in Fig. 2. A spherical AISI 304 steel sphere with $500 \mu \mathrm{m}$ diameter (1) was glued on a FemtoTools FT-S1000-LAT $(50 \mathrm{nN}-1000 \mu \mathrm{N})$ force sensor, and approached from a IPL-780 resin $2.1 \mathrm{~mm}$ diameter perturbing cylinder (3). Since the perturbation arises from the free surface deformation by the perturbing particle (characterized by the capillary charge), equivalence can be achieved by considering equal capillary charge $Q_{i}$ for both situations. The cylinder diameter was selected such that the resulting lateral capillary force $F_{\text {LatCap }}$ could be in the range of the force sensor.

Fig. 3 compares equation (1) with the experimental lateral capillary force $F_{\text {LatCap }}$ as a function of the obstacle-particle distance $r_{\text {obst-part }}$. As foreseen by the model assumptions, the theoretical model provides a good estimate for obstacleparticle distances larger than $2 \mathrm{~mm}$, and is indeed inaccurate for smaller distances. Since the particle is unlikely to be closer from disturbing neighbors than these $2 \mathrm{~mm}$, the following

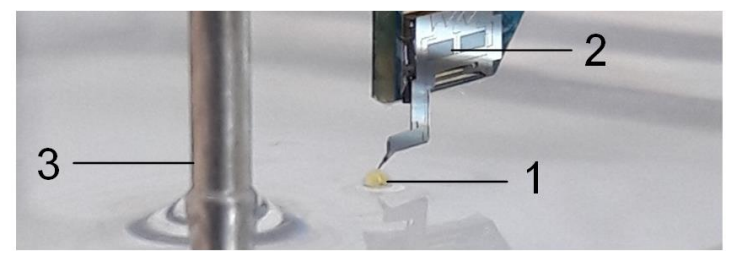

Fig. 2: Force measurement setup: a spherical steel sphere with $500 \mu \mathrm{m}$ diameter (1) was glued on a force sensor (2), and approached from a perturbing cylinder (3)

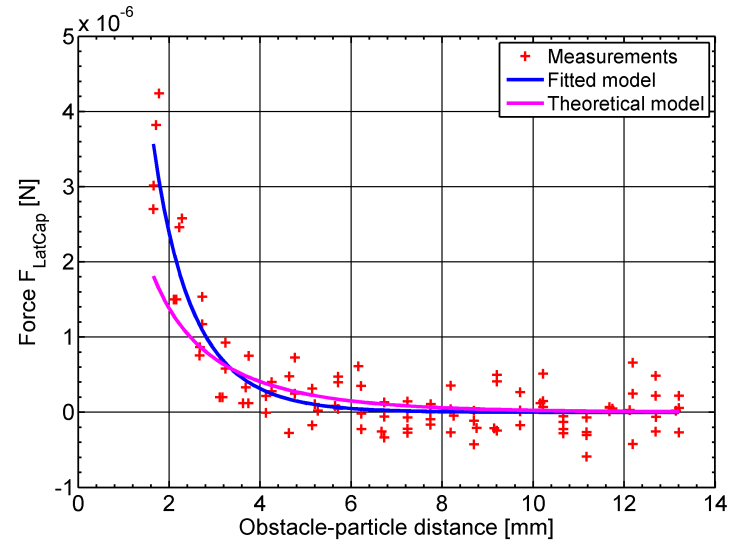

Fig. 3: Lateral capillary forces between particles. Each measured point represents the average of 50 continuous signals coming from the force sensor. The fitted model has been introduced to better estimate the force at small obstacleparticle distance, for which equation (1) is expected not to be accurate.

empirical fitted model was used to design the feedforward controller:

$$
F_{\text {LatCap }}=9.9 \cdot 10^{-6} \mathrm{~N}_{1}\left(800.56 \mathrm{~m}^{-1}\left\|\mathbf{X}_{\text {obst-part }}\right\|\right)
$$

where $\mathbf{X}_{\text {obst-part }}$ is the center-to-center obstacle-particle vector [m] and the resulting force $F_{\text {LatCap }}$ is given in $\mathrm{N}$.

Nevertheless, the thermocapillary actuation force generated by the laser was shown to be about $10 \mathrm{nN}$ [15], which is much lower than the disturbing lateral capillary force $F_{\text {LatCap }}$ at small obstacle-particle distances (at $2 \mathrm{~mm}$, the force is still about $2.37 \mu \mathrm{N}$ ). Therefore in the following, the particle will only be controlled at distances larger than $10 \mathrm{~mm}$. According to the model, two $500 \mu \mathrm{m}$ diameter steel spheres could be manipulated, provided the minimum distance between them should be larger than $4.7 \mathrm{~mm}$, distance at which the $F_{\text {LatCap }} \approx$ $9.95 \mathrm{nN}$. This limitation could be removed by increasing the laser power.

\section{Control Strategy with an Obstacle}

\section{A. Feedback Controller w/o Perturbation Disturbance}

The thermocapillary flows actually push particles. Therefore, particle velocity is likely to deviate from the current target direction (Fig. 4). Based on the measured particle position

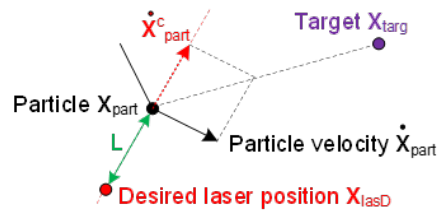

Fig. 4: Based on the measured particle position and velocity, a new laser position is calculated to realign the particle velocity towards the target. 


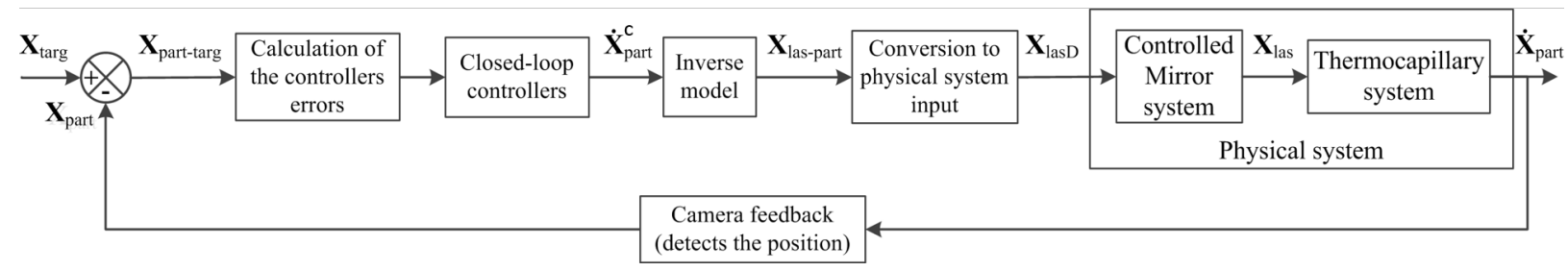

Fig. 5: Simplified schematic representation of the control scheme.

$\mathbf{X}_{\text {part }}$ and particle velocity $\dot{\mathbf{X}}_{\text {part }}$, the closed loop controller shown in Fig. 5 computes the velocity correction $\dot{\mathbf{X}}_{\text {part }}^{\mathrm{c}}$ to realign the particle velocity towards the target. As shown in [17], $\dot{\mathbf{X}}_{\text {part }}^{\mathrm{c}}$ depends on the laser-particle distance, so that the inverse model of this empirical characteristics provides a new laser-particle distance, and therefore the targeted laser position $\mathbf{X}_{\text {lasD }}$. The Controlled Mirror System can then be commanded for the targeted laser position to be set. As shown in [18], the transfer function of the Controlled Mirror system is considered to be equal to identity so only the control of the thermocapillary system has to be recalled here.

As represented in Fig. 7, the particle velocity magnitude and direction are actually decoupled, leading to the so-called steady-state particle velocity magnitude $\hat{\dot{r}}_{\text {part }}$ QSs and direction $\theta_{\text {corr }}$. The targeted laser position $\mathbf{X}_{\mathrm{las}}$ is obtained from the inverse model of the empirical characteristics [17] $\left(r_{\text {las-part }}\right.$ in $\mathrm{mm}, \hat{\dot{r}}_{\text {part Qss }}$ in $\mathrm{mm} \mathrm{s}^{-1}$ ) :

$$
r_{\text {las-part }}=-0.732 \hat{\dot{r}}_{\text {part QSS }}+4.177
$$

The orientation $\theta_{\text {las-part }}$ of the laser-particle vector $\mathbf{X}_{\text {las-part }}$ is then computed based on the correction angle $\theta_{\text {corr }}$

$$
\theta_{\text {las-part }}=\theta_{\text {part-targ }}-\theta_{\text {corr }}
$$

where $\theta_{\text {part-targ }}$ is the desired orientation, the orientation of the particle-target vector $\mathbf{X}_{\text {part-targ }}$.

From the laser-particle distance $r_{\text {las-part }}$ and laser-particle orientation $\theta_{\text {las-part }}$, the laser-particle relative position $\mathbf{X}_{\text {las-part }}$ is computed in the "Vector Comp" block (see Fig. 7). The corresponding desired laser position $\mathbf{X}_{\text {lasD }}=\mathbf{X}_{\text {part }}-\mathbf{X}_{\text {las-part }}$ is then computed.

\section{B. Feedforward Controller w/ Perturbation}

A feedforward controller (green box in Fig. 7) has been added to the system, to reject the disturbance of the lateral capillary force. As sketched in Fig. 6, the disturbing capillary force is calculated with (2) from the obstacle-particle distance. This disturbing force $F_{\text {LatCap }}$ is then converted into a disturbing velocity using the so-called Stokes' Law (commonly used to compute a viscous force from a particle velocity, according to [19]-[21]). In other words, an equivalent velocity $\hat{\dot{r}}_{\text {part }}$ QSS FF is computed, which is proportional to the disturbing force:

$$
\hat{\dot{r}}_{\text {part QSS FF }}=\frac{F_{\text {LatCap }}}{6 \pi \mu R_{\text {part }}}
$$

where $\mu$ is the liquid dynamic viscosity and $R_{\text {part }}$ the particle radius.

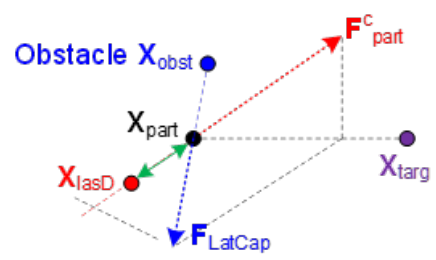

Fig. 6: Based on the measured particle and obstacle positions, a disturbing force $F_{\text {LatCap }}$ is computed from (2) and converted into a disturbing velocity from (5), which can be compensated with an appropriate laser position to realign the particle velocity towards the target.

Beside the disturbance magnitude, a correction angle $\theta_{\text {corr FF }}$ is also computed in the "FF Direction action" block of Fig. 7 using the following relation:

$$
\theta_{\text {corr FF }}=\theta_{\text {part-targ }}-\theta_{\text {obst-part }}
$$

This equation represents the direction opposite on which the lateral capillary force is acting w.r.t. the particle-target direction $\theta_{\text {part-targ. }}$.

Consequently, we see that this feedforward controller computes an estimated particle velocity at quasi steady-state $\hat{\dot{r}}_{\text {part QSS FF }}$ and a correction angle $\theta_{\text {corr FF }}$ (FF denotes the values of the inputs computed by the feedforward controller) based on the measured obstacle-particle position $\mathbf{X}_{\text {obst-part }}$. The system inputs computed by the feedforward and feebdack controllers are then added, as shown in Fig. 7 Using these resulting values, a desired laser position $\mathbf{X}_{\text {lasD }}$ is computed using the linearization procedure recalled in the previous subsection.

To define the feedforward controller, an inversion based approach was used that enabled to define $\hat{\dot{r}}_{\text {part }}$ QSS FF which is computed in the "FF Mag action" block (green box in Fig. 77). This block has as input the obstacle-particle distance $r_{\text {obst-part }}$ which is used to compute the lateral capillary force $F_{\text {LatCap }}$ acting on the particle using eq. 2. The inputs computed by both controllers are added in the "Input combinator" block as if they were defining a vector with polar coordinates: $\hat{\dot{r}}_{\text {part }}$ QSS xx and $\theta_{\text {corr xx }}$, according to equations (7, 8) on page 4

The resulting $\hat{\dot{r}}_{\text {part }}$ QSS (once limited to $4.5 \mathrm{~mm} / \mathrm{s}$ in the saturation block) and $\theta_{\text {corr }}$ are then used to compute a desired laser position $\mathbf{X}_{\text {lasD }}$. The latter one allows reaching the goals of the feedback and feedforward controllers. Indeed, for particles close to the obstacle (Fig. 8 a), the capillary force $F_{\text {LatCap }}$ 


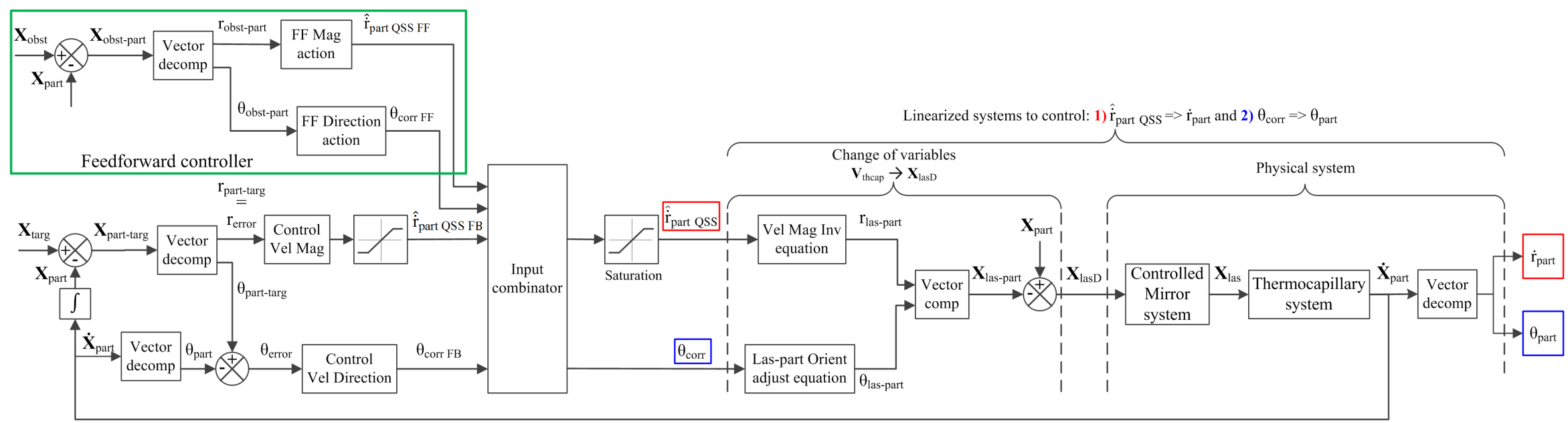

Fig. 7: Block diagram representation of the entire closed-loop system with the feedforward controller. This controller has two outputs: an estimated particle velocity

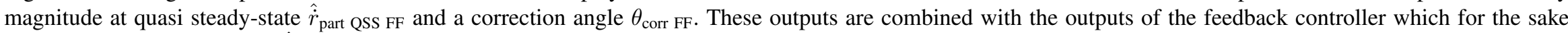

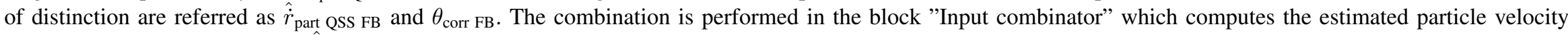
magnitude at quasi steady-state $\hat{\dot{r}}_{\text {part QSS }}$ and the correction angle $\theta_{\text {corr FB }}$ which are used to compute the desired laser position $\mathbf{X}_{\text {lasD }}$.

$$
\begin{aligned}
\hat{\dot{r}}_{\text {part QSS }} & =\sqrt{\hat{\dot{r}}_{\text {part QSS FB }}^{2}+\hat{\dot{r}}_{\text {part QSS FF }}^{2}+2 \hat{\dot{r}}_{\text {part QSS FB }} \hat{\dot{r}}_{\text {part QSS FF } \cos \left(\theta_{\text {corr FF }}-\theta_{\text {corr FB }}\right)}} \\
\theta_{\text {corr }} & =\theta_{\text {corr FB }}+\arctan \left(\frac{\hat{\dot{r}}_{\text {part QSS FF }} \sin \left(\theta_{\text {corr FF }}-\theta_{\text {corr FB }}\right)}{\hat{\dot{r}}_{\text {part QSS FB }}+\hat{\dot{r}}_{\text {part QSS FF }} \cos \left(\theta_{\text {corr FF }}-\theta_{\text {corr FB }}\right)}\right)
\end{aligned}
$$


is very strong and the feedforward controller dominates the feedback controller (whose goal is to take the particle to the target location $\mathbf{X}_{\text {targ }}$ ). Therefore, the desired laser position $\mathbf{X}_{\text {lasD }}$ generates a total radial force $F_{\mathrm{dr} \sigma \mathrm{r}}$ counteracting the

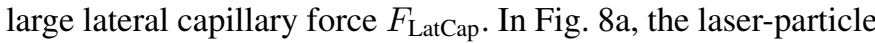
vector $\mathbf{X}_{\text {las-part }}$ is almost aligned with the lateral capillary force $F_{\text {LatCap. }}$ Conversely, for particles far away from the obstacle (Fig. 8p), the capillary force $F_{\text {LatCap }}$ is small and the feedback controller dominates the feedforward controller. Therefore, the desired laser position $\mathbf{X}_{\text {lasD }}$ leads to a total radial force $F_{\mathrm{dr} \sigma} \mathrm{r}$ displacing the particle towards the target location $\mathbf{X}_{\text {targ }}$. That is why in Fig. 8p, the laser-particle vector $\mathbf{X}_{\text {las-part }}$ is almost aligned with the particle-target vector $\mathbf{X}_{\text {part-targ. }}$. Note well that we considered here the case of repelling particles only.

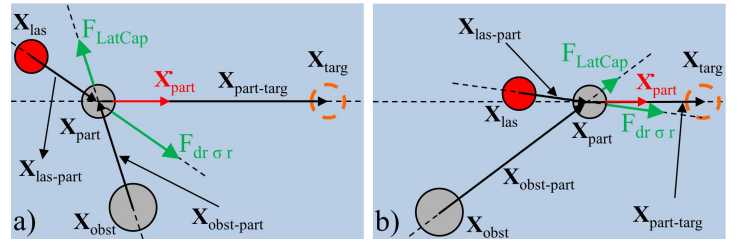

Fig. 8: (a) For small particle-obstacle distance, the FF action dominates and leads to a force $F_{\mathrm{dr} \sigma \mathrm{r}}$ mainly counteracting the large lateral capillary force $F_{\text {LatCap }}$; (b) For larger particleobstacle distance, the FB action dominates and leads to a resulting force $F_{\mathrm{dr} \sigma} \sigma$ pushing the particle towards the target.

\section{RESUlts}

\section{A. Simulation Results}

The control strategy was first tested in simulation using the Matlab/Simulink software. The starting and target positions of the sphere are respectively $(0,-15) \mathrm{mm}$ and $(0,20) \mathrm{mm}$ (fig. 9). The disturbing cylinder is located in $(7,0) \mathrm{mm}$.

The model for the particle velocity magnitude $\dot{r}_{\text {part }}$ can be seen as a cascade of a non-linear steady-state characteristics and a linearly time-variant dynamics. The first one is given by

$$
\hat{\dot{r}}_{\text {part QSS }}=26.086 \exp \left(-1.433 r_{\text {las-part }}\right)
$$

The second one links the steady-state output $\hat{\dot{r}}_{\text {part }}$ QSs to the particle velocity $\dot{r}_{\text {part }}$, according to the following second-order transfer function:

$$
\operatorname{VelDyn}(s) \equiv \frac{\dot{r}_{\text {part }}}{\dot{\hat{r}}_{\text {part QSS }}}=\frac{52.027}{s^{2}+7.6458 s+52.0274}
$$

The feedforward controller was first turned off. We can see in Fig. 9a that the laser primarily aims at pushing the particle towards the target location. Consequently, the $\mathrm{X}$-axis deviation can reach up to $1.22 \mathrm{~mm}$ in the worst case. The feedforward controller was then turned on to counteract the effect of the lateral capillary force $F_{\text {LatCap }}$ while also displacing the particle towards the target location. As a result (Fig. $9 \mathrm{~b}$, the particle is only deviated by a distance of $29 \mu \mathrm{m}$ in the Xaxis. The lateral capillary force $F_{\text {LatCap }}$ could however not be counteracted completely, because of saturation of the actuator.

\section{B. Experimental Results}

Experimental results were obtained with a AISI 304 steel $500 \mu \mathrm{m}$ diameter particle floating on distilled water in a plastic container placed above a white LED panel improving the particle position acquisition in real time with a camera. The laser is moved by a mirror using piezoelectric actuators [17]. As already devised, the perturbation is produced with a fixed IPL-780 resin cylinder ( $2.1 \mathrm{~mm}$ diameter, produced by Nanoscribe Photonics GT). The start and target points have the same $Y$ coordinate approximately only because it is not possible to control exactly the initial position of the floating particle. On its trajectory to the target, the closest point from the cylinder is at $12.8 \mathrm{~mm}$. As shown in Fig. 10, a maximum deviation of $3.05 \mathrm{~mm}$ is observed without the feedforward while it has been reduced to $1.27 \mathrm{~mm}$ with the feedforward activated.

\section{Discussion AND CONCLUSIONS}

This work opens perspectives towards parallel manipulation of multiple floating particles as long as they are not too close to each other, despite the intrinsic unstable physical principle. Indeed, due to the significant interaction capillary force, the actuator is saturated, which is accounted for by introducing a modle of this saturation in the controller. A more powerful laser would probably create a stronger surface tension gradient, and therefore allow the system to be faster. Of course, the main limitation of this work is that the (single) disturbing object is fixed. A next step would be to test the controller having two free moving particles. The capillary dipole interaction is indeed a 2D analogue of the Coulomb law in electrostatics [16]. Therefore, different forces arising from different particles could be added to estimate the force acting on a given particle. If these disturbing particles are fixes (=obstacles), the control scheme is probably still valid. If they are however free to move as well, the action of the laser beam must be distributed among the different particles to control, and this probably requires a modification of the control scheme. Another perspective would be to mitigate the strong capillary force with another force field. For instance, magnetic repulsion could reduce the capillary attraction.

\section{ACKNOWLEDGMENT}

Supported by 7/38 MicroMAST IAP (Belspo, Belgium).

\section{REFERENCES}

[1] A. Oulmas, N. Andreff, and S. Régnier, "3 d closed-loop motion control of swimmer with flexible flagella at low reynolds numbers," 2017 IEEE/RSJ International Conference on Intelligent Robots and Systems (IROS), pp. 1877-1882, 2017.

[2] V. Gauthier, A. Bolopion, and M. Gauthier, "Analytical formulation of the electric field induced by electrode arrays: Towards automated dielectrophoretic cell sorting," Micromachines, vol. 8, no. 8, 2017.

[3] Q. Zhou, V. Sariola, K. Latifi, and V. Liimatainen, "Controlling the motion of multiple objects on a Chladni plate," Nature Communications, vol. 7, p. 12764, Sep. 2016.

[4] M. Yin, E. Gerena, C. Pacoret, D. S. Haliyo, and S. Régnier, "Highbandwidth $3 \mathrm{~d}$ force feedback optical tweezers for interactive biomanipulation," 2017 IEEE/RSJ International Conference on Intelligent Robots and Systems (IROS), pp. 1889-1894, 2017. 


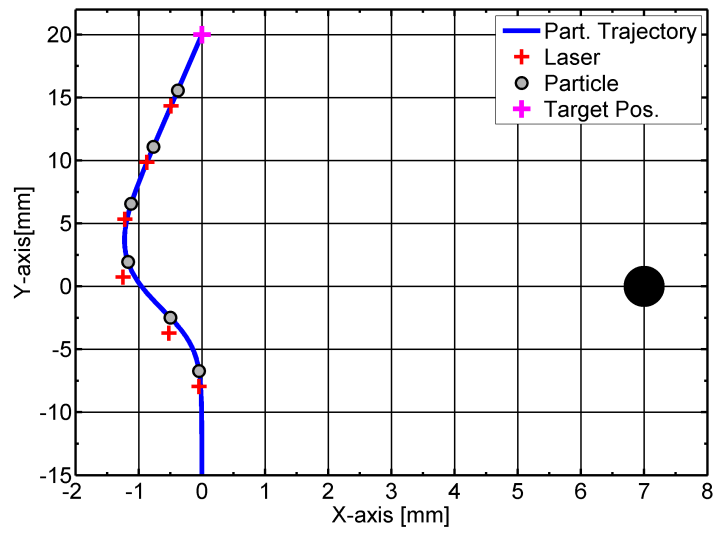

(a) Feedforward controller turned off

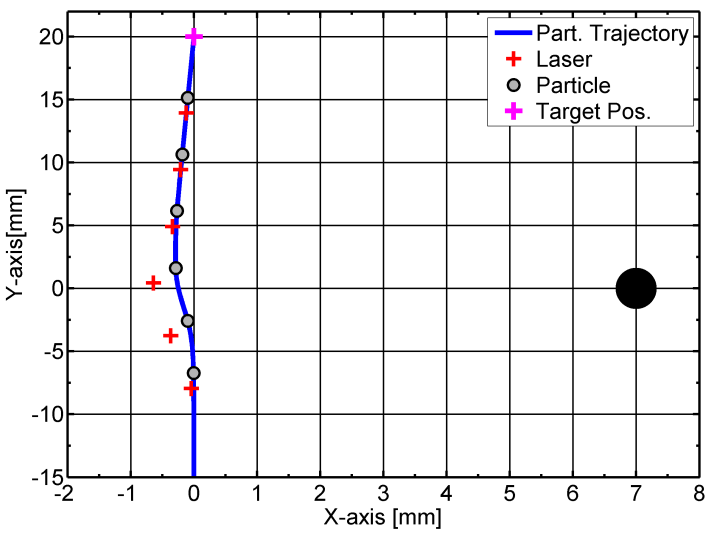

(b) Feedforward controller turned on

Fig. 9: Simulation results of a controlled manipulation of a $500 \mu \mathrm{m}$ diameter steel sphere in presence of a cylindrical obstacle. To show the effect of the controllers, the laser and particle positions are plotted for the time instants $t=2,3,4,5,6,7 \mathrm{~s}$. Note: the particle is only displaced towards a target location, no trajectory tracking is performed.

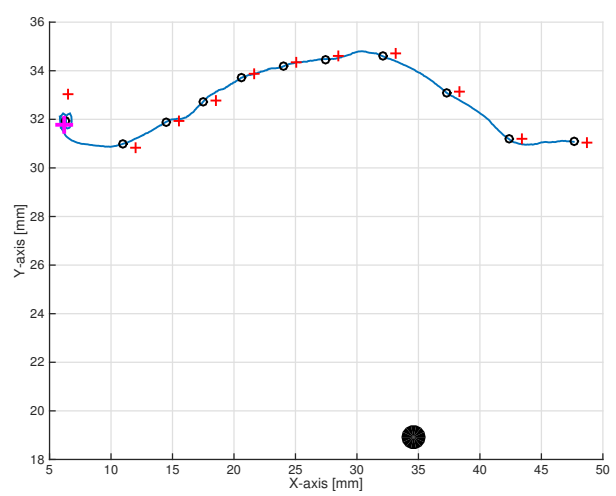

(a) Feedforward controller turned off

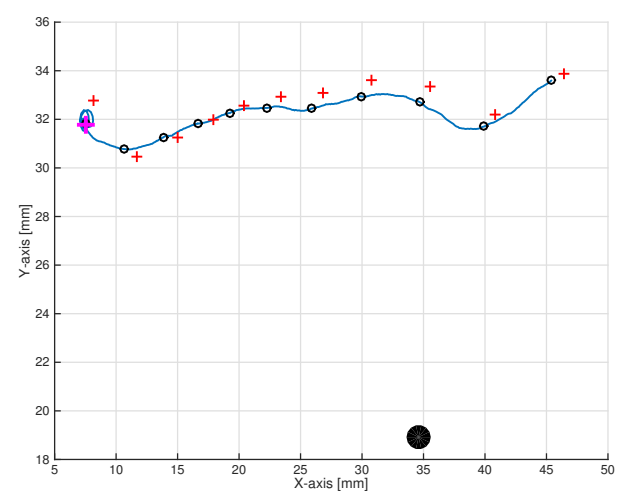

(b) Feedforward controller turned on

Fig. 10: Step response of the particle in the presence of an obstacle. The particle starts at the right of the cylinder and is controlled towards a target position (magenta cross) with a $\mathrm{Y}$ coordinate similar to its origin. The laser and particle positions are plotted for the time instants $t=0,3,6,9,12,15,18,21,24,27,30 \mathrm{~s}$.

[5] C. Pawashe, S. Floyd, and M. Sitti, "Multiple magnetic microrobot control using electrostatic anchoring," Applied Physics Letters, vol. 94, no. 16, p. 164108, 2009.

[6] E. Diller, S. Floyd, C. Pawashe, and M. Sitti, "Control of Multiple Heterogeneous Magnetic Microrobots in Two Dimensions on Nonspecialized Surfaces," IEEE Transactions on Robotics, vol. 28, no. 1, pp. 172-182, Feb. 2012.

[7] J. Zemnek, T. Michlek, and Z. Hurk, "Feedback control for noise-aided parallel micromanipulation of several particles using dielectrophoresis," ELECTROPHORESIS, vol. 36, no. 13, pp. 1451-1458, Jul. 2015.

[8] D. Wong, E. B. Steager, and V. Kumar, "Independent control of identical magnetic robots in a plane," IEEE Robotics and Automation Letters, vol. 1, no. 1, pp. 554-561, Jan 2016.

[9] S. Chowdhury, A. Thakur, P. Łvec, C. Wang, W. Losert, and S. K. Gupta, "Automated manipulation of biological cells using gripper formations controlled by optical tweezers," IEEE Transactions on Automation Science and Engineering, vol. 11, no. 2, pp. 338-347, April 2014.

[10] E. Vela, C. Pacoret, S. Bouchigny, S. Regnier, K. Rink, and A. Bergander, "Non-contact mesoscale manipulation using laser induced convection flows," in Intelligent Robots and Systems (IROS), 2008, pp. 913 918.

[11] E. Vela, "Non-contact microscale manipulation using laser-induced convection flows," Ph.D. dissertation, Universite Pierre et Marie Currie,
2010.

[12] E. Munoz, J. Quispe, P. Lambert, A. Bolopion, R. Terrazas, S. Régnier and E. Vela, "Optimizing the speed of single infrared-laser-induced thermocapillary flows micromanipulation by using design of experiments," J Micro-Bio Robot, vol. 12, pp. 65-72, 2017.

[13] W. Hu, Q. Fan, and A. T. Ohta, "Interactive actuation of multiple opto-thermocapillary flow-addressed bubble microrobots," Robotics and Biomimetics, vol. 1, no. 1, pp. 1-6, 2014.

[14] _ "An opto-thermocapillary cell micromanipulator," Lab Chip, vol. 13, no. 12, pp. 2285-2291, 2013.

[15] R. T. Mallea, "Thermocapillary micromanipulation: laser induced convective flows towards controlled handling of particles at the free surface," Ph.D. dissertation, Université libre de Bruxelles / Université de FrancheComté, 122017.

[16] P. A. Kralchevsky and K. Nagayama, Chapter 7 - Lateral Capillary Forces between Partially Immersed Bodies, ser. Studies in Interface Science. Elsevier, 2001, vol. 10, pp. $287-350$.

[17] R. T. Mallea, A. Bolopion, J.-C. Beugnot, P. Lambert, and M. Gauthier, "Laser-induced thermocapillary convective flows: A new approach for non-contact actuation at microscale at the fluid/gas interface," IEEE Transactions on Mechatronics, 2017.

[18] _ - "Closed-loop particle motion control using laser-induced thermo- 
capillary convective flows at the fluid/gas interface at micrometric scale," 2018.

[19] R. A. Flynn, A. L. Birkbeck, M. Gross, M. Ozkan, B. Shao, M. M. Wang, and S. C. Esener, "Parallel transport of biological cells using individually addressable $\{$ VCSEL $\}$ arrays as optical tweezers," Sensors and Actuators B: Chemical, vol. 87, no. 2, pp. 239 - 243, 2002.

[20] S. Chowdhury, P. Svec, A. Thakur, C. Wang, W. Losert, and S. K. Gupta, "Enhancing range of transport in optical tweezers assisted microfluidic chambers using automated stage motion," in ASME 2013 International Design Engineering Technical Conferences and Computers and Information in Engineering Conference, 2013, p. V001T09A031.

[21] M.-C. Zhong, X.-B. Wei, J.-H. Zhou, Z.-Q. Wang, and Y.-M. Li, "Trapping red blood cells in living animals using optical tweezers," Nature Communications, vol. 4, p. 1768, 2013. 